\title{
Telecommunication Jitter Measurement in the Transmultipexer Systems Method Based on the Wavelet Pattern Correlation
}

\author{
Jan Borgosz and Bogusław Cyganek \\ Electronic Engineering and Computer Science Department, \\ Academy of Mining and Metallurgy, Mickiewicza 30, 30-059 Kraków, Poland \\ \{borgosz, cyganek\}@agh.edu.pl
}

\begin{abstract}
The paper presents a new method of the measure of the jitter in telecommunication. Jitter is the phenomenon which is especially harmful to the transmultiplexer systems, so its exact measure is very important to the system engineer. Described scheme is based on the recognition and correlation of the patterns generated by means of the wavelet transform. This research is a continuation of the work on development of the wavelet based jitter measurement methods that do not use the reference clock. Presented algorithm allows to estimate the jitter parameters in relation to the jitter wavelet base functions. Practical computer algorithm is also presented. Additionally, such topics like the wavelet type, its order, as well as calibrating methods are also discussed. This paper extends previous research and gives not only a proposal but also implementation details.
\end{abstract}

\section{Introduction}

A jitter is an unwanted, spurious transmission clock phase modulation that originates from the physics of semiconductor device [6][11].

Estimating the jitter of a transmission clock is an important problem in telecommunication measurements, especially for the transmultiplexer systems. Each transmultiplexer is time to a frequency-domain multiplexer and may be treated as a discrete multitone modulator (DMT) [1]. It is common knowledge that DMT blocks are fundamental for the Digital Subscriber Line (DSL) technology so presented solution seems to be interesting for DSL engineers and designers.

The classic approach to the jitter measurement analysis usually consists of processing steps that use a reference clock [6, 11, [12]. The most troublesome part of the measurement process is to correlate slopes of the reference and received clocks. The purpose of this paper is to present a totally different wavelet based approach to jitter measurement analysis as compared to the aforementioned methods. Presented approach extends the research work which was presented during the ICWAA 2001 conference [5]. 
Jitter test equipment is used with an Equipment Under Test (EUT). Generated test signals are transmitted over telecom line into the Equipment Under Test. The EUT retransmits received data to the meter over a telecom line. Test equipment processes all received information and calculates results [3] 3].

In the classic approach, the implementation of the jitter meter requires on the reference clock.

\section{Mathematical Representation of Jitter}

A jitter as an unwanted, spurious transmission clock phase modulation. This phenomenon can be modeled using a modulation scheme, which allows us to describe it with the multitone technique [3][10]. A case of single tone modulation can be described as follows:

$$
y(t)=A \cdot \cos \left(2 \cdot \pi \cdot f_{\text {clock }} \cdot t+\varphi_{m}(t)\right),
$$

where $y$ is the jittered clock signal with amplitude $A[\mathrm{~V}]$ and base frequency $f_{\text {clock }}[\mathrm{Hz}]$. The phase modulating function $\varphi_{m}(t)$ can be described as follows:

$$
\varphi_{m}(t)=2 \cdot \pi \cdot k \sin \cdot\left(2 \cdot \pi \cdot f_{j i t t} \cdot t\right)
$$

where $f_{\text {jitt }}$ is jitter frequency [Hz], $k \geq 0 \wedge k \in R$ the jitter amplitude in telecommunication UI units (UI means Unit Interval which is equal to one cycle of transmission clock). All calculations will be presented for the sine function which easily can be changed to other wave shapes. Note that the sine waves after the comparator will be square wave with $50 \%$ duty cycle - ideal clock signal [7][11][ [12].

A single tone modulated signal can be written as follows:

$$
y_{P M}(t)=A \cdot \cos \left(\Omega \cdot t+\Delta \Theta_{P M} \cdot \sin \varpi \cdot t\right) .
$$

As shown in [10] this representation can be replaced by a more appropriate form that makes use of the Bessel function:

$$
y_{P M}(t)=A \cdot \sum_{n=-\infty}^{\infty} J_{n}\left(\Delta \Theta_{P M}\right) \cdot \cos (\Omega+n \cdot \varpi) \cdot t,
$$

where $J_{n}$ is an n-th order Bessel function of the first kind. In this case equations (1) and (2) can be rewritten as follows:

$$
y(t)=A \cdot \sum_{n=-\infty}^{\infty} J_{n}(2 \cdot \pi \cdot k) \cdot \cos \left(\Omega+n \cdot \varpi_{j}\right) \cdot t,
$$

where $\Omega=2 \cdot \pi \cdot f_{\text {clock }}$ and $\varpi_{j}=2 \cdot \pi \cdot f_{\text {jitt }}$. 


\section{Preprocessing of the Jittered Signal}

A jittered sine signal can be integrated by a circuit with a much higher cut-off frequency than the maximum frequency in the input signal. In this case, the integrator is a kind of an accumulator. A jittered signal given by (5) after the integration is equal to:

$$
y_{I N T}(t)=\frac{A}{\Delta T} \cdot \int_{t}^{t+\Delta T} \sum_{n=-\infty}^{\infty} J_{n}(2 \cdot \pi \cdot k) \cdot \cos \left(\Omega+n \cdot \varpi_{j}\right) \cdot t,
$$

where $\Delta T$ is the integration period. Because a direct analysis of (6) is somewhat cumbersome, therefore some numerical computations were performed and are presented in Fig. 1a, 1b. Numerical computations were performed for square waves as well - sine waves after the comparator are presented in Fig. 1c, 1d. Note, that there is no need for the reference clock for detection of jitter. This is a confirmation of the presented here calculations $[5]$.

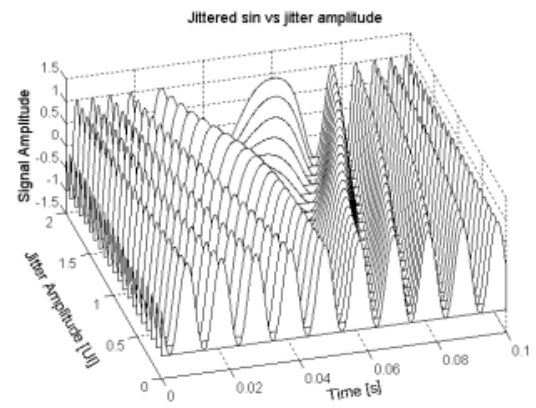

(a)

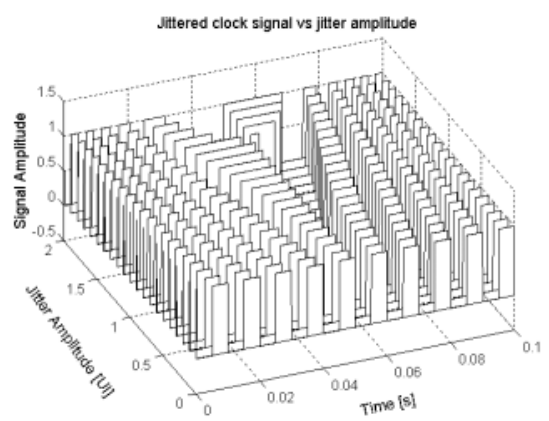

(c)

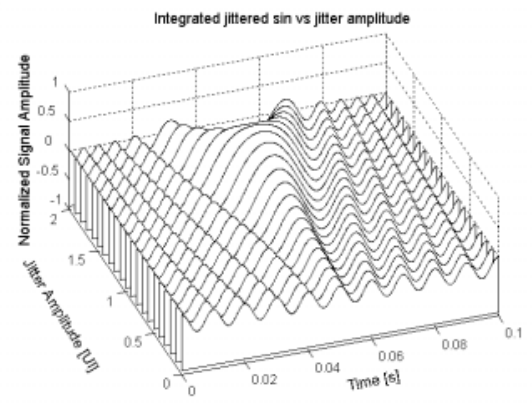

(b)

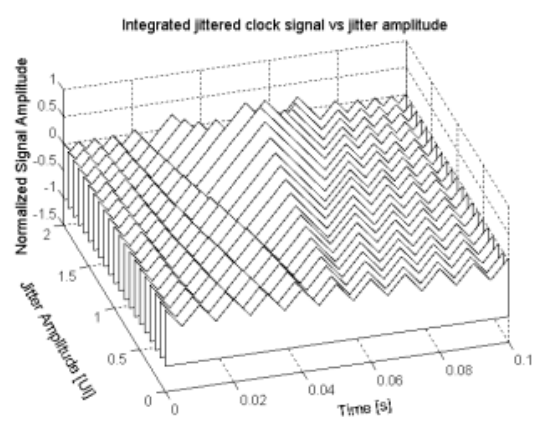

(d)

Fig. 1. a) Set of jittered sine waves with jitter frequency $f_{\text {jitt }}=10 \mathrm{~Hz}$, carrier frequency $f_{n}=100 \mathrm{~Hz}$ for different jitter amplitudes, b) the same sine waves after integration, c) set of jittered clock signals with jitter frequency $f_{\text {jitt }}=10 \mathrm{~Hz}$, carrier frequency $f_{n}=100 \mathrm{~Hz}$ for different jitter amplitudes, d) the same clock signals after integration. 


\section{Wavelet Transform Applied to Jitter Analysis}

High quality jitter measurements involve measurements of an amplitude and frequency of signals, as well as their change in time. In authors opinion, the best tool for such an analysis is the Continuous Wavelet Transform (CWT) [1] 8] which is represented by the following equation:

$$
C(s, p)=\int_{-\infty}^{\infty} f(t) \cdot \Psi(s, p, t) d t
$$

where $\Psi(s, p, t)$ is a mother wavelet, $s$ the scale and $p$ the position. Inserting (6) into (7) provides us to the following formula:

$$
C(s, p)=\int_{-\infty}^{\infty}\left(\left(\frac{A}{\Delta T} \cdot \int_{t}^{t+\Delta T}\left(\sum_{n=-\infty}^{\infty} J_{n}(2 \cdot \pi \cdot k) \cdot \cos \left(\Omega+n \cdot \varpi_{j}\right) \cdot t\right) d t\right) \cdot \Psi(s, p, t)\right) d t
$$

As can be observed in (8), there is no easy way to find a relationship between jitter parameters and $C W T$ coefficients for a signal after integration 9]. There are two basic problems:

1) wavelet selection

2) finding correlation of wavelet coefficients $C(s, p)$ with jitter parameters (i.e. its amplitude and frequency).

The basic idea was to find other approach which allows to eliminate problems listed above. Each calculation can be done in an absolute or a relative way. Due to the troubles with the solution of the problem by the absolute way, we decide to investigate the relative one. Our idea was to use jittered signal with well known jitter parameters - called the "jitter base". We try to find relation between any jittered signal and jitter base and find unknown jitter parameters from the relation which has been found. Of course linear one would be the best.

\section{Relational Jitter Measurement}

The new idea to jitter analysis is to employ relative measurements. We can relate the two matrices $A$ and $B$, by means of the following equation:

$$
A \cdot r=B \text {. }
$$

In real situations we rather attempt to solve for $r$ in a sense of a minimization problem:

$$
\min _{r}(B-A \cdot r) \text {. }
$$

For the purpose of this paper we solve (10) by means of the least squares method. We introduce a notation presented below: 


$$
r=\operatorname{LSQR}(A, B),
$$

which means that $r$ will be found with the least squares method.

In all of the following cases the matrix (vector) $A$ will be called "the base" and matrix (vector) B will be called "the test". So in all measurements relation $r$ will be searched which allows to transform "base" space to the "test" space. A selection of spaces will be discussed in the next sections. We have checked all possibilities of the spaces generation (results are presented below), to show that our choice is not accidental.

\subsection{Relation between Integrated Jittered Signals}

First attempt was to check relation between non transformed signals in a time domain that are in the form of integrals (6). Due to this supposition, $A$ will be a base vector generated as an integrated jittered signal with known jitter parameters, and $B$ will be a test vector, for which relation $r$ will be searched. Numerically calculated exemplary relation $r$ for the varying test vector is presented in Fig.2a. It can be seen that there is no easy relation between $r$ and changing jitter parameters, thus it is not clear how the two spaces can be related.

\subsection{Relation between CWT of the Jittered Signals}

Due to a fail of the first method, the next attempt was made based on the observation that if jitter parameters are changed, then jittered signal "changes scale". It can be observed also that increasing jitter amplitude results in something like a local stretching of the signal. A similar conclusion may be drawn for situation with a changing jitter frequency. In this case $A$ will be a base matrix generated as an CWT coefficients of the jittered signal with known jitter parameters, and $B$ will be a test matrix with CWT coefficients of the jitter function with the other parameters, for which relation $r$ will be searched. Numerically calculated exemplary relation $r$ for the varying test vector parameters is presented in Fig.2b. Results are not very promising, like in the previous case.

\subsection{Relation between CWT of the Integrated Jittered Signals}

In this case the measure space was selected as the CWT of the integrated jittered signal. It can be observed that in this case, increasing jitter amplitude results in something like changing parameters of the standing wave. Similar conclusion may be done for changing jitter frequency. In this case $A$ will be a base matrix generated as an CWT coefficients of the integrated jittered signal with known jitter parameters and $B$ will be a test matrix with CWT coefficients of the integrated jitter function with the other parameters for which relation $r$ will be searched. Numerically calculated 
exemplary relation $r$ for the varying test vector parameters is presented in Fig.2c. This way obtained results are very interesting. Some linear relation may be observed between the jitter parameters and the CWT jitter base. Detailed discussion of this phenomenon will be presented in the next section.

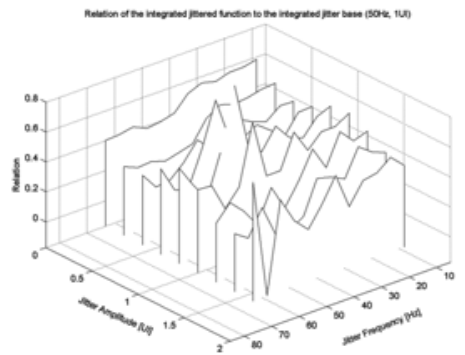

(a)

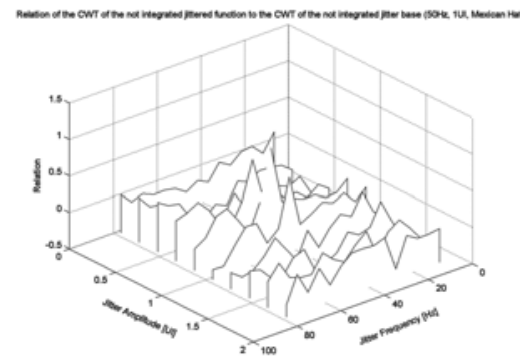

(b)

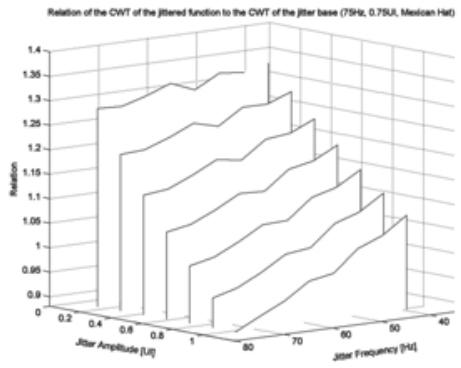

(c)

Fig. 2. a) Relation $r$ for the time domain jitter signals, b) relation $r$ for the varying jitter parameters and for the CWT jitter space, c) Relation $r$ in respect to the varying jitter parameters for the CWT of the integrated jitter signal - Mexican Hat wavelet $(75 \mathrm{~Hz}, 0.75 \mathrm{UI})$

\section{Relational Jitter Measurement with Wavelet Base of the Integrated Signal}

\subsection{Selection of the Wavelet Type}

Due to discussion presented in previous sections and experiments which were described in the ICWAA 2001 paper [5], bases with CWT of the integrated jitter signal with different wavelet types were checked. 
The following wavelets were tested:

- Mexican Hat,

- Morlet,

- Coiflets 2-5,

- Biorthogonal 2.6 2.8 4.4 5.5 6.8,

because their shapes appeared to be the most appropriate to analyze the signals shown in Fig.4b and Fig.4c (see section 4.1.). Exemplary results are presented only for the Biorthogonal 4.4, Morlet and Mexican Hat - see Fig.3.

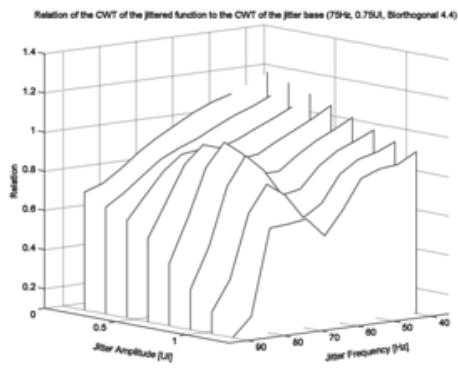

(a)

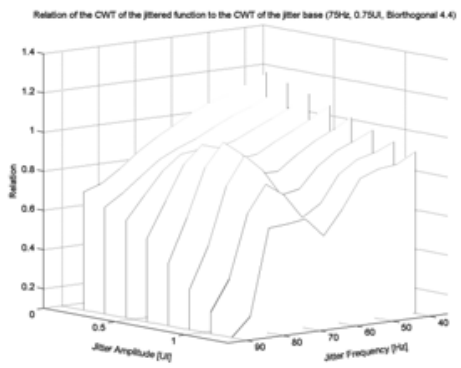

(c)

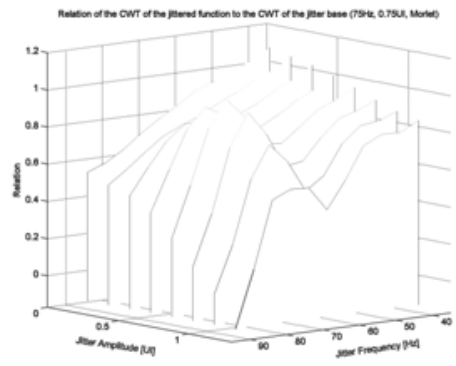

(b)

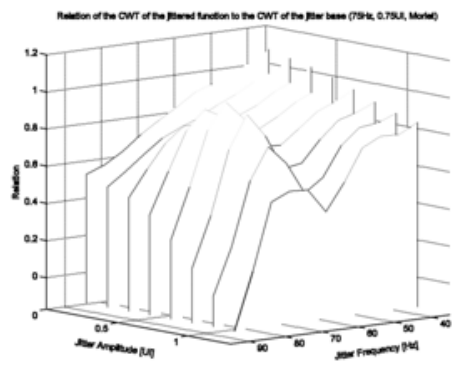

(d)

Fig. 3. a) Relation $r$ for the varying jitter parameters and for the CWT of the integrated jitter space - Biorthogonal 4.4 wavelet, b) relation $r$ for the varying jitter parameters and for the CWT of the integrated jitter space - Morlet wavelet, c) relation $r$ for the varying jitter parameters and for the CWT of the integrated jitter signal - Mexican Hat wavelet $(100 \mathrm{~Hz}$, $0.25 \mathrm{UI}), \mathrm{d}$ ) Relation $\mathrm{r}$ for the varying jitter parameters and for the CWT of the integrated jitter signal - Mexican Hat wavelet $(50 \mathrm{~Hz}, 1 \mathrm{UI})$

Only the Mexican Hat wavelet gives almost linear relation, when in other cases parabolic or other relations were obtained. Thus, there is a first important remark that a wavelet type used in base function is significant and decides about type of relation. 
Presented results show that the Mexican Hat wavelet gives the best results, due to nearly linear characteristic of $r$. Set of the numerical tests were done which confirmed our observations.

Figure 4 depicts relation $r$ in the 2D-style plot. It may be observed that relation $r$ may be described by the classic linear equation $(y=a x+b)$, where parameters $a$ and $b$ depend on jitter frequency - this can be written as follows:

$$
r=a\left(f_{j i t t}\right) \cdot A_{j i t t}+b\left(f_{j i t t}\right) .
$$

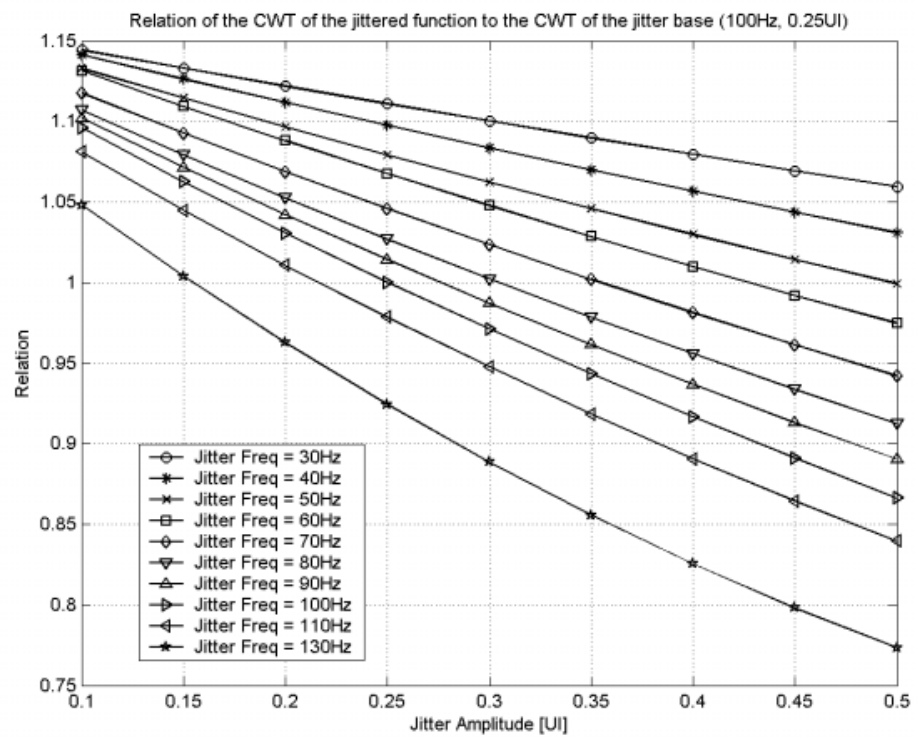

Fig. 4. Relation $r$ for the varying jitter parameters and for the CWT of the integrated jitter signal - Mexican Hat wavelet

\subsection{Relation between CWT of the Integrated Jittered Signals - Analytic Analysis}

Results obtained by means of the numerical analysis may be verified by the analytic relations. Let us assume that:

$$
C(s, p)=\int_{-\infty}^{\infty} f(t) \cdot \Psi(s, p, t) d t=C W T(f(t), \Psi) .
$$


The relation $r$ may be rewritten as follows:

$$
r=\operatorname{LSQR}\left(C W T\left(y_{I N T}(t), \Psi\right), C W T\left(y_{B A S E_{-} I N T}(t), \Psi\right)\right),
$$

where:

$$
\begin{gathered}
y_{I N T}(t)=\frac{A}{\Delta T} \cdot \int_{t}^{t+\Delta T} \sum_{n=-\infty}^{\infty} J_{n}(2 \cdot \pi \cdot k) \cdot \cos \left(\Omega+n \cdot \varpi_{j}\right) \cdot t, \\
y_{B A S E_{-} I N T}(t)=\frac{A_{B A S E}}{\Delta T} \cdot \int_{t}^{t+\Delta T} \sum_{n=-\infty}^{\infty} J_{n}\left(2 \cdot \pi \cdot k_{B A S E}\right) \cdot \cos \left(\Omega+n \cdot \bar{\varpi}_{j B A S E}\right) \cdot t .
\end{gathered}
$$

Thus, we can rewrite (16) as follows:

$$
r=\frac{A}{A_{B A S E}} \cdot \operatorname{LSQR}\left(\begin{array}{l}
C W T\left(\int_{t}^{t+\Delta T} \sum_{n=-\infty}^{\infty} J_{n}(2 \cdot \pi \cdot k) \cdot \cos \left(\Omega+n \cdot \varpi_{j}\right) \cdot t, \Psi\right) \\
C W T\left(\int_{t}^{t+\Delta T} \sum_{n=-\infty}^{\infty} J_{n}\left(2 \cdot \pi \cdot k_{B A S E}\right) \cdot \cos \left(\Omega+n \cdot \varpi_{j B A S E}\right)\right.
\end{array}\right.
$$

\section{Conclusions}

In this paper we conclude that a jitter measurement may be realized as calculation of the relation between $C W T$ coefficients of integrated jittered signals and CWT coefficients of a base jitter signal.

This method provides a novel way of jitter measurement without usage of the reference clock. An introduction to the analytic description of the method was presented. Different types of wavelets were analyzed in a context of their application to the presented method. However, only wavelets with the Mexican Hat mother functions produce sought linear results.

There are still subjects that need further investigation, however. These are:

- detailed analytic solution of the problem,

- analytic solution including wavelet type,

- detailed analysis of the range of jitter parameters change.

Presented solution due to its innovatively may be very interesting for the modern telecommunication business associated with DSL and transmultiplexer techniques, which has to look for the new techniques which allow to use cheap hardware resources (like twisted-pair telephone lines) to deliver modern multimedia to the client. 
Acknowledgments. This paper is a result of research work registered in The State Committee for Scientific Research of Poland (KBN) number 4 T11D 00523 and its publication is sponsored by means of the KBN founds.

\section{References}

1. Akansu A., Duhamel P., Lin X., Courville M.: Orthogonal transmultiplexers in communication: A review. IEEE Trans. SP, vol. 46 (1998) 979-995

2. Białasiewicz J.: Wavelets and Approximations (in Polish). WNT (2000)

3. Borgosz J., Cyganek B., Korohoda P.: Jitter Telecom Test Equipment Based On FPGA Structure, International Conference on Signals and Electronic Systems ICSES'2001, Łódź, Poland (2001) $397-402$

4. Borgosz J., Cyganek B.: Digital Implementation of Jitter Generator for Telecom Test Equipment, Digital Signal Processing for Multimedia Communications and Services, EURASIP Conference: ECMCS 2001, Budapest, Hungary (2001)

5. Borgosz J., Cyganek B.: A Proposal of Jitter Analysis Based on a Wavelet Transform, The Second International Conference on Wavelet Analysis and Its Applications ICWAA01 Hong Kong Baptist University, Hong Kong (2001) 257-268

6. Feher and Engineers of Hewlett-Packard: Telecommunication Measurements Analysis and Instrumentation, Hewlett-Packard (1991)

7. Glover I. A., Grant P.M.: Digital Communications. Prentience Hall (1991)

8. Prasad L., Iyengar S.S.: Wavelet Analysis. CRC Press (1997)

9. Starck J.-L., Murtagh F., Bijaoui A.: Image Processing and Data Analysis. The Multiscale Approach. Cambridge University Press, (2000)

10. Szabatin J.: Foundations of Signals Theory (in Polish). WKL (2000)

11. Trischitta P.R., Varma E.L.: Jitter in Digital Transmission System. Artech House Publishers (1989)

12. Takasaki Y., Personick S.D.: Digital Transmission Design and Jitter Analysis. Artech House Publishers (1991) 